\title{
A Survey on Geometric Correction of Satellite Imagery
}

\author{
Chintan P. Dave \\ I.T. Department \\ Parul Institute of Engineering \\ and Technology, Vadodra, \\ Gujarat, India
}

\author{
Rahul Joshi \\ I.T. Department \\ Parul Institute of Engineering \\ and Technology, Vadodra, \\ Gujarat, India
}

\author{
S. S. Srivastava, $\mathrm{PhD}$ \\ Scientist-SE, \\ MSDPD/DPSG/SIPA \\ SAC, ISRO, Ahmedabad, \\ Gujarat, India
}

\begin{abstract}
In remote sensing data, geometric distortions are observed due to acquisition system and the movements of the platform. A geometric correction of the image is required whenever the image is to be compared with existing maps or with other images. This review paper enlists various Preprocessing techniques for geometric correction of raw images. Preprocessing of satellite image is prerequisite for further analysis applications such as change detection, object identification, image classification etc.
\end{abstract}

\section{Keywords}

Geometric Distortion, Pre-processing, Geometric Correction, Ortho-rectification, Image Rectification, Image Registration, Mathematical Model, Ground Control Points

\section{INTRODUCTION}

Remote Sensing is the science and art of retrieving information about objects without physically or intimate contact with them. There are two major characteristics of image i.e. Geometric and Radiometric. Geometric characteristics based on co-ordinates of image and the radiometric characteristics describe the actual information content in image. Distortion is noise in information or alternation of original signal or shape. Raw images generally contain such significant amount of distortion that they cannot be used directly with map base products such as geographic information system (GIS) [1]. These distortions may be due to several factors including the perspective of the sensor optics; the motion of the scanning system; the motion of the platform; the platform altitude, attitude, and velocity; the terrain relief; and, the curvature and rotation of the Earth. The sources of geometric distortions are classified in two categories: Observer and Observed.

Preprocessing of satellite images prior to image application is essential. Preprocessing commonly encompasses a series of sequential operations, such as atmospheric correction (normalization), image registration, geometric correction, radiometric correction and masking [5]. This review paper is mainly focus on geometric correction of distorted raw image. The goal of geometric correction is to make image distortion free. Image registration is the process of spatially aligning two or more images scene obtained at different time or from different sensors. The word image registration is used in different literatures as geometric registration, and rectification of geometric distortion or polynomial affine transformation. The image acquired earlier date is known as base image and, recently acquired image with error is to be corrected.

\section{GEOMETRIC DISTORTION}

Remote sensing imagery contains unique geometric distortion. The sources of distortion can be grouped into two broad categories: the Observer or the acquisition system (platform, imaging sensor and other measuring instruments etc.) and the Observed (atmosphere and Earth) [1]. Table 1: describes in more detail the sources of distortion for each category.

Table 1 Sources of Geometric Distortions

\begin{tabular}{|c|l|l|}
\hline \multirow{4}{*}{ Category } & Sub-category & \multicolumn{1}{c|}{ Description } \\
\hline \multirow{4}{*}{$\begin{array}{l}\text { The Observer } \\
\text { or acquisition } \\
\text { system }\end{array}$} & Platform & $\begin{array}{l}\text { Variation of movement, } \\
\text { attitude }\end{array}$ \\
\cline { 2 - 3 } & Sensor & $\begin{array}{l}\text { Variation in sensor } \\
\text { mechanism }\end{array}$ \\
\cline { 2 - 3 } The Observed & $\begin{array}{l}\text { Measuring } \\
\text { instrument }\end{array}$ & Time variation \\
\hline & Atmosphere & $\begin{array}{l}\text { Atmospheric conditions: } \\
\text { Refraction, reflection, } \\
\text { turbulence }\end{array}$ \\
\cline { 2 - 3 } & Earth & $\begin{array}{l}\text { Curvature, rotation, } \\
\text { topographic effect }\end{array}$ \\
\cline { 2 - 3 } & Map & Ellipsoid/Datum \\
\hline
\end{tabular}

Geometric distortion may cause changes of scale over the image, irregularities in the angular relationships among the image elements, displacement of objects in an image and occlusion of one image element by another. There are two types of geometric error occurrences $[4,5]$ :

\subsection{Systematic Errors}

Generally caused by Scan skew, mirror scans, velocity variance, panoramic distortion, platform velocity and, earth rotation. These errors can correct through analysis of system characteristics and ephemeris.

\subsection{Nonsystematic Errors}

Generally caused by altitude variance and varied platform attitude. These errors can correct with the use of ground control points.

Geometric distortions can also categorize as internal distortions and external distortions. Internal distortions are generally occurs due to platform or observer's movement, while external distortions are due to observed object. Following figure shows the various effects generated during image distortions. 


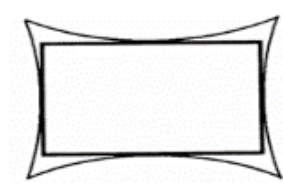

(A) Radial distortion

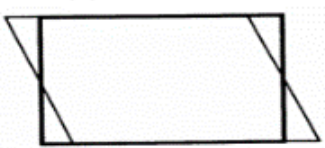

(E) Skew

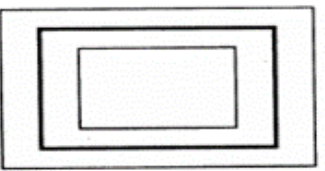

(C) Scale error

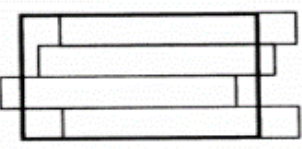

(G)Step-wise distortion

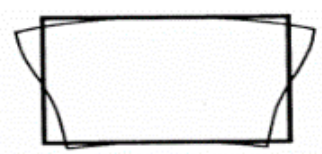

(B) Tangential distortion

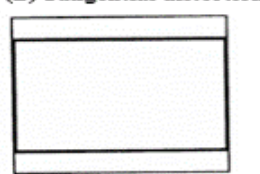

(F) Along track scan error

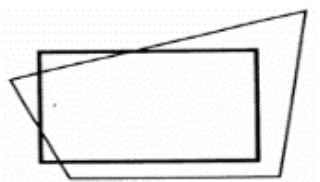

(D) Projection error

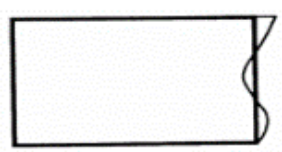

(H)Scan-Line Scale error
Fig 1: Internal Distortions

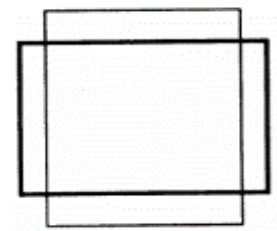

(c) $\mathrm{V} / \mathrm{H}$ error

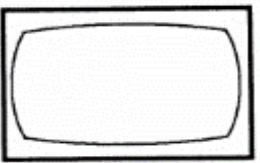

(g)Distortion due to Earth curvature

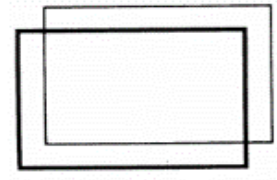

(a)Shift error

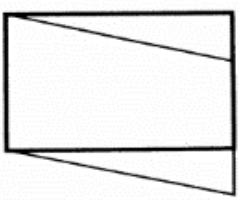

(e)Skew of scan line

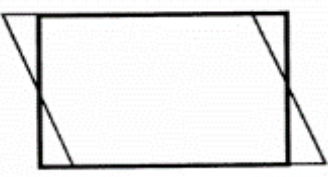

(d)Skew

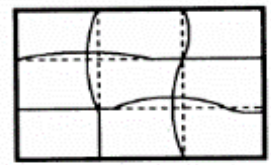

(h)Terrain relief Displacement

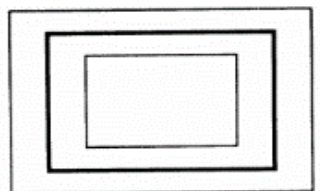

(b) Scale error

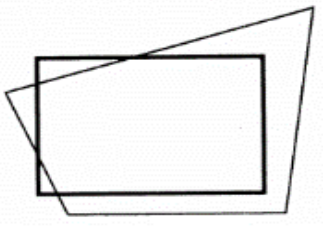

(f)Projection distortion
Fig 2: External Distortions

\section{GEOMETRIC CORRECTION}

Geometric corrections are made to correct the inconsistency between the location coordinates of the raw image data, and the actual location coordinates on the ground or base image. Several types of geometric corrections include system, precision, and terrain corrections. Geometric correction is necessary to preprocess remotely sensed data and remove geometric distortion so that individual picture elements (pixels) are in their proper planimetric (x,y) map locations [2]. Planimetric elements in geography are those features that are independent of elevation, such as roads, building footprints, and rivers and lakes. They are represented on two-dimensional maps as they are seen from the air, or in aerial photography. Geometrically corrected imagery can be used to extract accurate distance, polygon area, and direction (bearing) information.

Remotely sensed imagery collected from airborne or space borne sensors often contain internal and external geometric errors. These can be systematic (predictable) or nonsystematic (random) as listed above. Some of these errors can be corrected by using ephemeris of the platform and known internal sensor distortion characteristics. Other errors can only be corrected by matching image coordinates of physical features recorded by the image to the geographic coordinates of the same features collected from a map or global positioning system (GPS). To remove the error from image rectification is necessary. This process is known as an image rectification.

There are four different levels of geometric correction of remotely sensed imagery [5]:

a) Registration- alignment of one image to another image of the same area

b) Rectification- alignment of image to a map so that the image is planimetric, just like the map; Also known as geo-referencing

c) Geocoding- A special case of rectification that includes scaling to a uniform standard pixel GIS

d) Orthorectification- Correction of the image, pixel by pixel for topographic distortion.

There are two major techniques for geometric correction [5]:

a) Modeling

First technique is modeling the nature and magnitude of all sources of geometric distortion and find the corresponding correction formulae. This approach works well when all sources of distortion are well understood and can be quantified. This is not the case for all sources of distortion, particularly not for the unsystematic variations in satellite altitude etc.

\section{b) Empirical}

Empirical approach uses reference points (ground control points). This approach required some ancillary information such as image acquisition data, yaw, pitch, roll values and other information in XML format.

There are two major image geo-correction techniques.

a) Image-to-Map geo-correction- this method used reference frame such as toposheets, maps or any standard (Universal Transverse Mercator) reference images. 


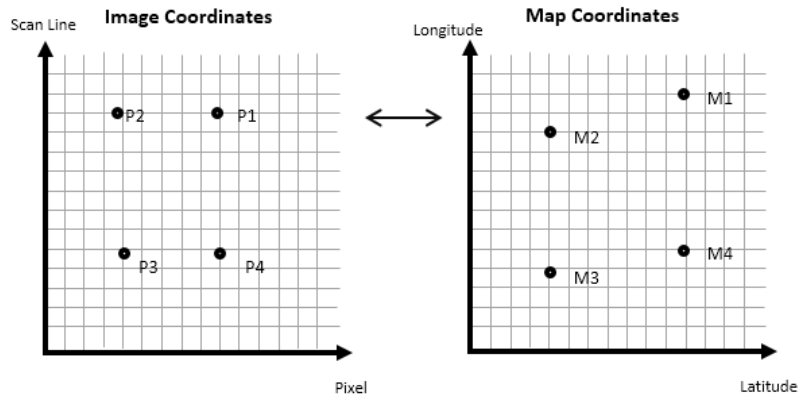

Fig 3: Image-to-Map geo-correction

b) Image-to-Image geo-correction- these method match one image to another so the same geographic area is positioned coincident with respect to the other. This type of geometric correction is used when it is not necessary to have each pixel assigned a unique $(\mathrm{x}, \mathrm{y})$ coordinate in a map

\section{VIEWING GEOMETRIC MODEL}

A "viewing geometry model" consists of establishing a relation between any pixel $(1, \mathrm{p})$ of the level $1 \mathrm{~A}$ image and the relative point $(\lambda, \phi)$ on terrestrial reference system [8]. In this relation, the altitude $\mathrm{h}$ of the point on the ground is supposed to be known.

Fig 3: Viewing geometry model projection

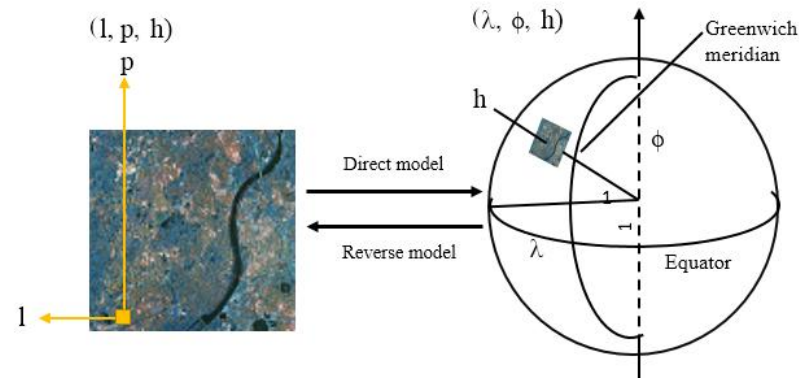

Where,

1 is a line value of image

$\mathrm{p}$ is a pixel value of image

$\lambda$ is a Longitude in geodetic reference system

$\varphi$ is a Latitude in geodetic reference system

$\mathrm{h}$ is an altitude during image acquisition

Direct model is to compute the intersection between the look directions of any pixel $(1, p)$ with an Earth model. This Earth model could be estimated by using a Digital Elevation Model (DEM) above an ellipsoid. Reverse model is to compute the intersection between the look direction $(\lambda, \varphi)$ of DEM elevated reference with a distorted image [8].

\section{MATHEMATICAL MODEL}

The mathematical distortion model such as polynomial model establish a correspondence between the global coordinates of the distorted image to those in reference image or map. Polynomial models usually needed for the transformation between image and object coordinates. This polynomial equation can calculate new output pixel locations $(x, y)$ and relate image location to the Ground Control Point location. The following 2D equations are used to commonly for the polynomial model [2].

$$
\begin{aligned}
& X=a+a_{1} x+a_{2} y+a_{3} x y \\
& Y=b+b_{1} x+b_{2} y+b_{3} x y
\end{aligned}
$$

\section{ORTHORECTIFICATION}

To maintain the geometry of a captured image even when the camera is not viewing the scene at the nadir angle, the images have been transformed by a process known as Orthorectification [4]. Ortho-rectification, converts an image obtained at an arbitrary roll-pitch-yaw of the camera to one as if obtained when roll, pitch, and yaw were all zero. Orthorectification stabilizes the camera as if it is always looking down and heading toward the north. Ortho-rectified images are easier to register and analyses because these are not geometrically distorted. Correction for an image geometry by orthorectification, however, results in some intensity distortions in the image caused by the resampling process.

\section{INTERPOLATION}

Once the applying geometric transformation; image may be shear, rotate, transformed or skewed. Pixel Interpolation is necessary for filling missing values of area. For that pixel brightness values must be determines. There may not be any direct one-to-one relation between base image and image which is rectified [5]. There is mechanism for determining the brightness value, this process is known as pixel interpolation.

\section{RESAMPLING}

Resampling process used to determine the digital values to place in the new pixel location of the corrected output image, this process known as a resampling. Resampling required to estimate a new pixel between existing pixels due to noninteger transformed (x, y) [3]. Table 2 shows types of resampling method.

Table 2. Types of Resampling Method

\begin{tabular}{|c|l|}
\hline Type & \multicolumn{1}{c|}{ Description } \\
\hline Nearest Neighbor & $\begin{array}{l}\text { New pixels value get form closest } \\
\text { pixel of old pixel }\end{array}$ \\
\hline $\begin{array}{c}\text { Bilinear } \\
\text { Interpolation }\end{array}$ & $\begin{array}{l}\text { New pixels value calculated from the } \\
\text { weighted average of } \\
4(2 \times 2) \text { nearest pixels }\end{array}$ \\
\hline Cubic Convolution & $\begin{array}{l}\text { New pixels are computed from } \\
\text { weighting } 16(4 \times 4) \\
\text { surrounding DNs }\end{array}$ \\
\hline
\end{tabular}

\section{COMPLICATIONS IN GEOMETRIC CORRECTION}

Following are the major difficulties occurred during geometric correction process.

1. Selection of the feature points from the images

2. Determination of correspondence between images

3. Selection of right transformation function that can represents geometric distortion between images

4. No technique to determine correspondence between features points when the images have nonlinear geometric distortions.

\section{CONCLUSION}

Every remote sensing satellite need and requirements of geometric correction process. This process may applied on pre-launch phase or post-launch phase. This paper is only review the geometric correction techniques, work may remain for radiometric correction. Once derived mathematical model may use for identifying further relationship between images. This paper only enlist and provide overview of various geometric correction parameters, but one can further take following references for more details. 


\section{ACKNOWLEDGMENTS}

I am very thankful to Dr. Shailendra Shanker Srivastava to help me during this survey. I am also thankful Rahul Joshi for his immense help in paper preparation and submission.

\section{REFERENCES}

[1] Noam Levin, "Fundamentals of Remote Sensing", Elsevier Publications, 1999, pages "57-97"

[2] Lt. Dr. S. Santosh Baboo, Mr. Thirunavukkarasu, "Geometric Correction in High Resolution Satellite Imagery using Mathematical Methods: A Case Study in Kiliyar Sub Basin”, Global Journal of Computer Science and Technology: Graphics \& Vision", June-2014, volume-14, Global Journals Inc.(USA)-0975-4172

[3] A. L. Choo, Y. K. Chan, V. C. Koo, "Geometric Correction on SAR Imagery", Progress In Electromagnetics Research Symposium Proceedings, KL, MALAYSIA, March-2012

[4] Narayan Panigrahi, B.K. Mohan and G. Athithan, "Preprocessing Algorithm for Rectification of Geometric
Distortions in Satellite Images", Defence Science Journal, DRDO, Vol. 61, No. 2, March-2011

[5] Lt. Dr. S. Santosh Baboo, M. Renuka Devi, "Geometric Correction in Recent High Resolution Satellite Imagery: A Case Study in Coimbatore, Tamilnadu", International Journal of Computer Applications, January-2011, 09758887

[6] T. Toutin, "Review article: Geometric processing of remote sensing images: models, algorithms and methods", Natural Resources Canada, Canada Centre for Remote Sensing- 588 Booth Street, Ottawa, Ontario, K1A 0Y7, Canada, June-2010, Taylor \& Francis

[7] Jalal AMINI and Ali Reze Mohamadi HASHEMI, Iran, "Geometric Correction in Ikonos Images - Case Study: Tehran, Iran".

[8] Serge RIAZANOFF, "Spot Satellite Geometry Handbook", 31030-Toulouse, Cadex 4, France, 2002, edition 1 